\title{
Transcellular Activation of the Human Immunodeficiency Virus Type 1 Long Terminal Repeat in Cocultured Lymphocytes
}

\author{
ADRIANA MARCUZZI, ${ }^{1}$ JUDAH WEINBERGER, ${ }^{2}$ AND OFRA K. WEINBERGER ${ }^{1 *}$ \\ Department of Physiology and Cellular Biophysics ${ }^{1}$ and Departments of Medicine and Pharmacology, ${ }^{2}$ \\ Columbia University, New York, New York 10032
}

Received 17 January 1992/Accepted 27 March 1992

\begin{abstract}
One of the unexplained aspects of the progression of AIDS is that immunological abnormalities are detectable before CD4 $^{+}$T-helper cell depletion occurs (A. R. Gruters, F. G. Terpstra, R. De Jong, C. J. M. Van Noesel, R. A. W. Van Lier, and F. Miedema, Eur. J. Immunol. 20:1039-1044, 1990; F. Miedema, A. J. Chantal-Petit, F. G. Terpstra, J. K. M. E. Schattenkerk, F. de Wolf, B. J. M. Al, M. Roos, J. M. A. Lang, S. A. Danner, J. Goudsmit, and P. T. A. Schellekens, J. Clin. Invest. 82:1908-1914, 1988; G. M. Shearer, D. C. Bernstein, K. S. Tung, C. S. Via, R. Redfield, S. Z. Salahuddin, and R. C. Gallo, J. Immunol. 137:2514-2521, 1986). In this report, we describe a mechanism by which human immunodeficiency virus type 1 (HIV-1)-infected cells can influence neighboring HIV-1-infected $T$ lymphocytes and uninfected $T$ cells as well. We have examined the interaction of T-cell and macrophage cell lines that are transfected with HIV-1 DNA by using cocultured lymphocytes. The HIV-1 constructs we used lack a functional pol gene and therefore do not produce infectious virus. Cocultivation results in the transcellular activation of the HIV long terminal repeat in the cocultured T cells. This transcellular activation is evident in as little as $3 \mathrm{~h}$ of cocultivation, at ratios of HIV-expressing cells to target cells as low as 1:1,000, and is dependent on the Tat-responsive element. The demonstration that a small number of HIV-expressing cells can affect a large number of uninfected bystander cells in a short period of time suggests a mechanism by which global immune dysfunction can precede the high prevalence of infected cells.
\end{abstract}

Early in the course of human immunodeficiency virus type 1 (HIV-1) infection, before there is evidence of a significant viral load, widespread defects in the antigen-specific responses of $\mathrm{CD}^{+} \mathrm{T}$ cells have been reported. These immune defects can arise before a substantial decrease in the number of $\mathrm{CD}^{+}$cells becomes evident $(8,16,22,23)$. Thus the manifestations of disease do not appear to result solely from the activation of the latent provirus in infected cells but may also reflect the effects of chronically infected cells on the immune function of uninfected neighboring cells.

There is a growing body of evidence that demonstrates that exposure of lymphoid cells in vitro to various HIV-1 products (e.g., Tat and gp120) can result in functional alterations in the uninfected cells. It has been reported that soluble Tat peptide is taken up by cells in culture, either by adsorptive endocytosis (12) or possibly by an integrinrelated adhesion receptor (2), and can inhibit the antigeninduced proliferation of lymphocytes (26) and stimulate growth of a Kaposi's sarcoma cell line (6). Soluble gp120 can inhibit phytohemagglutin-induced lymphocyte proliferation (14), induces changes in interleukin- 2 and interleukin-2 receptor expression $(5,9,20)$, and induces interleukin-1 and prostaglandin production in monocytes (27). The large quantities of viral proteins needed to effect these cellular changes $(5,9,14,20,26,27)$, however, make it difficult to assess the role that soluble viral products play in the immunodeficiencies that are apparent in the early stages of infection.

We hypothesized that an infected cell might express either a cellular or a viral product that could transactivate a latent HIV long terminal repeat (LTR) in a nearby cell. To test this hypothesis, we established cell lines expressing specific HIV genes under the control of the HIV LTR. Because no infectious virus is produced in this system, it is possible to

${ }^{*}$ Corresponding author. assess the effect of HIV gene expression on adjacent uninfected cells.

\section{MATERIALS AND METHODS}

Cell lines and plasmids. Jurkat, MOLT 4, and CEM are human T-lymphoblastoid cell lines, and U937 is a human promonocytic cell line. The plasmids $\mathrm{pHXB} \Delta \mathrm{pol}(\mathrm{p} \Delta \mathrm{pol})$, pIIIextatpA (25), pIIIexE7(rev env) (24), and pHIVCAT (21) were provided by J. Sodroski. $\mathrm{p} \Delta$ pol was derived from the $\mathrm{HXBc} 2$ proviral clone and was generated by deleting a 700-bp fragment at the KpnI site (nucleotide 3829). This deletion affected only the pol coding region and consequently abrogated the reverse transcriptase activity of the virus. LTR-directed transcription was assayed by measuring chloramphenicol acetyltransferase (CAT) activity after transfection of the plasmid pHIVCAT. The pHIVAtarCAT plasmid (17) was provided by $\mathrm{M}$. Muesing and was generated by deleting a 40-bp SacI-HindIII fragment from the LTR. For the generation of stable cell lines, the plasmids $\mathrm{p} \Delta \mathrm{pol}$ neo $^{r}$ and pHIVCAT-neo ${ }^{r}$ were used. These plasmids were constructed by ligation of a 2.6-kb $\mathrm{BamHI} \mathrm{Neo}^{\mathrm{r}}$ cassette (SV2neo) into the HpaI site of $\mathrm{p} \Delta \mathrm{pol}$ or the BamHI site of pHIVCAT.

Transfections. Transient transfections were performed with DEAE-dextran, and stably transfected cell lines were generated by electroporation. After the selection of G418resistant cells, $\mathrm{p} \Delta$ pol-neo ${ }^{\mathrm{r}}$ was transfected transiently with pHIVCAT to identify clones which were positive for functional Tat expression. Similarly, HIVCAT-neo ${ }^{r}$ cells were transfected transiently with $\mathrm{p} \Delta$ pol (which supplied Tat), and clones which had integrated a functional CAT gene under the control of the HIV LTR were selected.

In experiments in which transiently transfected cells were used, the cells were cultured for $24 \mathrm{~h}$ after transfection with the indicated plasmid. After being washed, the cells were 


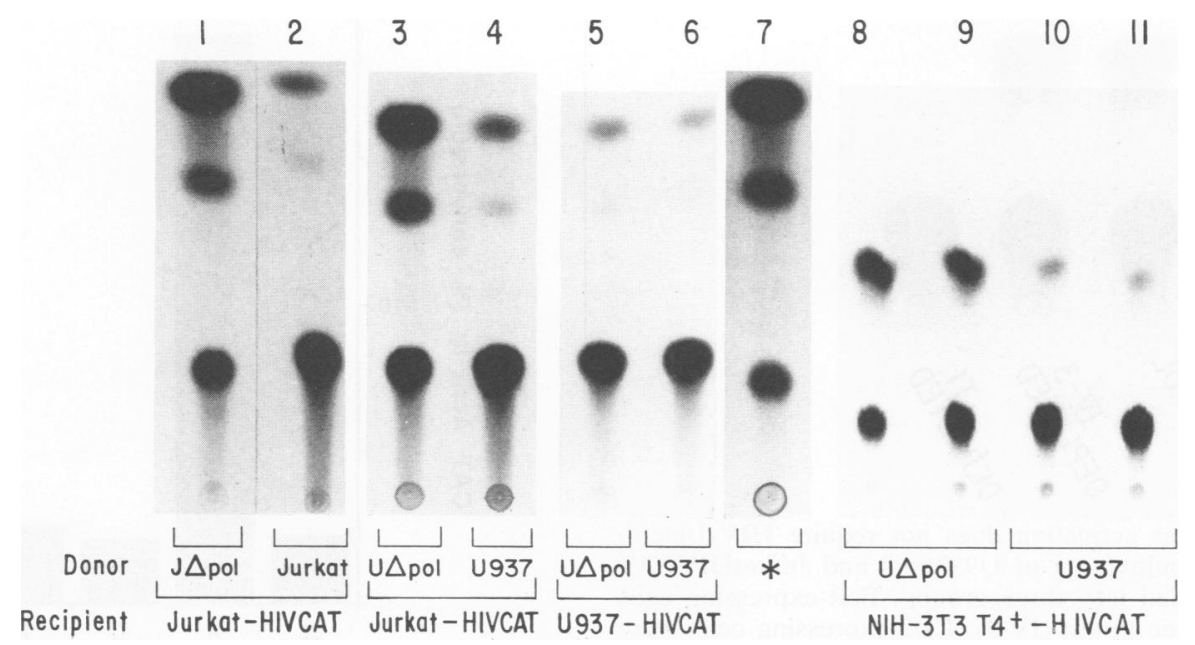

FIG. 1. Transcellular activation of the HIV LTR. pHIVCAT-transfected cells were cocultured with pHXB $\Delta$ pol-expressing cells or control cells for $48 \mathrm{~h}$, and the CAT activity was assayed. Lanes: $1,2,5$, and 6 , stably transfected cells; 3, 4, 8, and 9, transiently transfected cells; 7, CAT activity induced in U937HIVCAT after transiently transfecting them with pHXB $\Delta$ pol.

resuspended in fresh medium at a final cell density of $6 \times$ $10^{5} / \mathrm{ml}$. Cell extracts were prepared after $48 \mathrm{~h}$ of coculture and assayed for CAT activity. Cocultures of stable transfectants were started at a cell density of $4 \times 10^{5} / \mathrm{ml}$ and incubated, unless otherwise indicated, for $72 \mathrm{~h}$ prior to harvest. The IV-3 antibody is specific for FcRII and was provided by M. Fanger.

\section{RESULTS}

HIV gene expression induces transcellular activation of a naive population of cells. U937 and Jurkat cells were transfected with $\mathrm{pHXB} \Delta \mathrm{pol}$, a plasmid that was derived from the $\mathrm{HXBc} 2$ proviral clone by a $700-\mathrm{bp}$ deletion in the polymerase-coding region. This deletion results in a loss of reverse transcriptase activity, and therefore no infectious virus particles are produced in this system.

To determine whether transcellular activation occurs, pHXB $\Delta$ pol-transfected U937 and Jurkat cells were cocultured with indicator cells, i.e., cells transfected with an HIV-1 LTR-CAT plasmid (pU3R-III) (21). After $48 \mathrm{~h}$ of coculturing, the cells were harvested and assayed for CAT activity. When U937 $\Delta$ pol or Jurkat $\Delta$ pol cells were cocultured with JurkatHIVCAT cells, a dose-dependent transactivation was readily apparent, as indicated by increased expression of CAT activity (Fig. 1, lanes 1 and 3). This effect was not seen when JurkatHIVCAT was cocultured with U937 or Jurkat cells (lanes 2 and 4) or with U937 or Jurkat cells that were transfected with a control plasmid. The addition of chloroquine had no effect (data not shown).

The ability of HIV-1-expressing cells to activate bystander $\mathrm{T}$ cells was evident when $\mathrm{pHXB} \Delta$ pol was expressed in a variety of different cell lines: Jurkat, CEM, or MOLT 4 (T cell); Raji (B cell); K562; and U937 (monocyte) (data not shown). All tested T-cell lines, as well as NIH 3T3-T4 ${ }^{+}$cells (Fig. 1, lanes 8 to 11), were susceptible to activation. The data suggest that there is some cell type specificity with this transcellular activation; in U937HIVCAT cells cocultured with pHXB $\Delta$ pol-transfected cells, only trace amounts of CAT activity were detected (lanes 5 and 6 ). This is not due to nonfunctional LTR-CAT in the U937 cells, because transient transfection of $\mathrm{pHXB} \Delta \mathrm{pol}$ into these cells results in significant CAT activity (lane 7). THP-1, another monocytic cell line transfected with pHIVCAT, is also refractory to activation. These experiments were carried out with stably transfected cell lines as well as transiently transfected cells as both recipients and donors, and similar results were obtained.

We do not observe syncytia in our cultures after expression of this construct. Two-color fluorescent labeling of the cell populations did not reveal any cell fusion: $U \Delta$ pol cells were labeled with fluorescein, and JHIVCAT cells were labeled with rhodamine as described previously (10), and after the cells were cocultured for $48 \mathrm{~h}$, microscopic observation revealed no doubly stained cells (data not shown). Although cells transfected with $\mathrm{p} \Delta$ pol express immunoprecipitable cytoplasmic gp120, only low levels of gp120 are expressed on the cell surface (data not shown). If high-level gp120-expressing cells were generated by the transfections, these cells probably died early in the selection process. Thus, it is likely that fusion does not occur in our cultures because sufficient levels of gp120 are not expressed on the cell surface.

Even after expression of human CD4 murine cells do not permit HIV infection or multinucleated syncytium formation (11). The induction of the HIV LTR in murine NIH 3 T3 cells cocultured with U937Dpol cells (Fig. 1, lanes 8 to 11) suggests that these fusion events are not necessary for transcellular activation in these cultures. The level of induction of the LTR in NIH 3T3 cells was lower than the induction observed in human $\mathrm{T}$ cells, as has been reported for Tat-mediated activation of the HIV-1 LTR in rodent cells when both molecules are coexpressed (18).

Following cocultivation of U937 2 pol and JurkatHIVCAT cells, the cells were divided into three equal groups. Cells were treated with monoclonal antibodies specific for either U937 (IV-3, anti-FcR) or Jurkat (T1, anti-CD2) cells. By using magnetic beads coupled to anti-immunoglobulin $G$ (IgG), the IV-3-expressing cells were depleted from one group and the CD2-expressing cells were depleted from another group. If cells had undergone fusion and thus expressed both markers, or if expression of the cell surface markers had been modulated as a result of fusion, CAT activity would appear in both selected groups. In these 


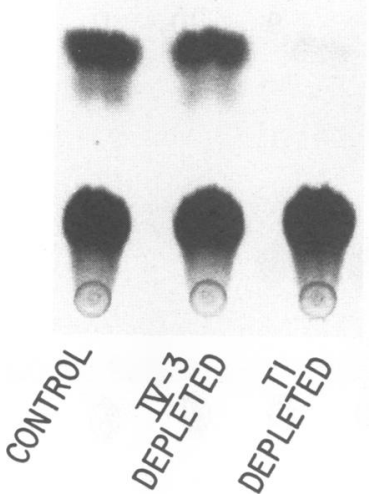

FIG. 2. Transcellular activation does not require HIV-1-mediated fusion. After cocultivation of U937 $\mathrm{pol}$ and JurkatHIVCAT cells, they were divided into three groups. FcR-expressing cells were depleted from one group (IV-3), CD2-expressing cells were depleted from another (T1), and then the CAT activity was assayed.

experiments, all the CAT activity is found in the T-cell population (JHIVCAT) and none is found in the U937 cell population (Fig. 2).

Transcellular activation is mediated by Tat. The primary identified viral gene product necessary for high-level activation of the HIV-1 LTR is Tat $(1,3,4,25)$, and Tat activation requires the TAR element in the $\operatorname{LTR}(17,21)$. To determine whether Tat is required for transcellular activation, we utilized two TAR deletion mutants of pHIVCAT. MOLT4 cells were individually transfected with pHIVCAT or pHIV $\Delta$ tarCAT (40-bp SacI-HindIII deletion) and then cocultured with U937 $\Delta$ pol cells. As shown in Fig. 3, greater than $95 \%$ of the CAT activity that resulted from cocultivation required integrity of the TAR region in the LTR expressed in the recipient cells. Similar results by using Jurkat $\Delta$ pol cells as donors or CEM and Jurkat cells as the recipients (data not shown) were obtained. The CD23 $\Delta S$ TAR mutant, with only a 4-bp deletion (+34 to +37$)(19)$ also did not respond to the transcellular activator. Transcellular activation, therefore, requires the presence of the specific TAR element, suggesting that the effect is mediated by Tat. However, the addition of anti-Tat antibody to the cocultivation medium had no effect on transactivation (data not shown).

To determine whether cell contact is necessary for this transactivation, the conditioned supernatant from U937 or U937 $\Delta$ pol cells growing at $10^{6}$ cells per ml was added to JHIVCAT cells at concentrations that varied from 25 to $75 \%$ of the final volume. The JHIVCAT cells were then cultured for 48 to $72 \mathrm{~h}$ at a density of $5 \times 10^{5}$ cells per ml. The LTRs in these cells were not transactivated (data not shown). Transactivation was also not detectable when the donor and recipient populations of cells were cultured without physical contact by separating them with a Millicell culture insert (Fig. 4). Positive controls for transactivation consisted of JHIVCAT cells cocultured directly with U937Dpol cells. These results suggest that either the mediator of the activation is highly unstable or transactivation requires cell-cell contact.

Parameters of transcellular activation. To determine the ability of small numbers of cells to effect LTR activation in bystander cells, various numbers of U937 pol cells were cocultured with the JurkatHIVCAT cells. Significant transactivation was observed over a wide range of ratios and was

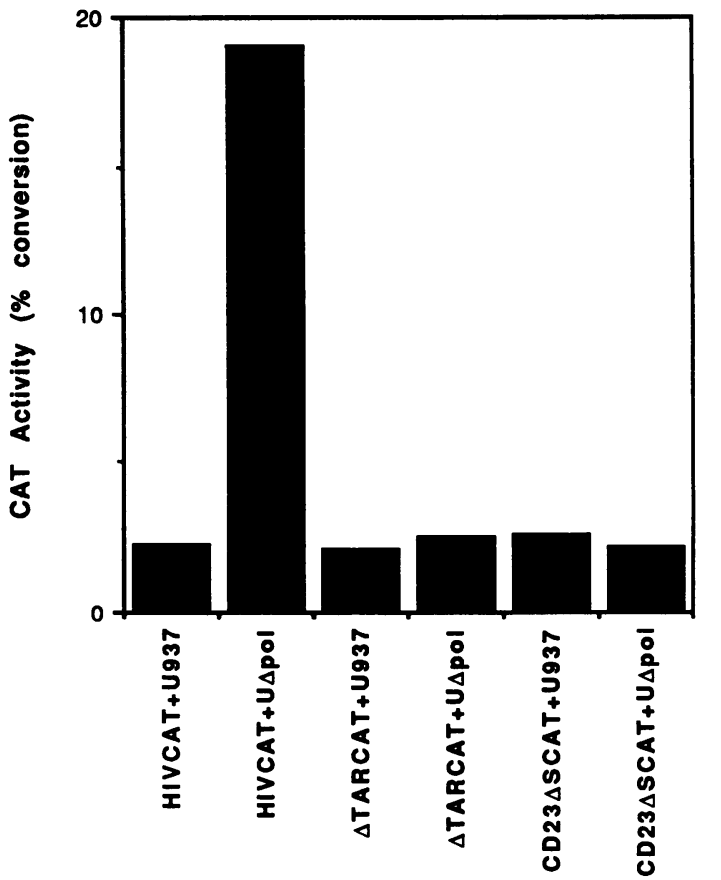

FIG. 3. Transcellular activation is mediated by Tat. Jurkat cells were transiently transfected with pHIVCAT, pHIV $\Delta \operatorname{tarCAT}$, or pHIVCD23 SCAT. These cells were then cocultured for $48 \mathrm{~h}$ with U937 $\mathrm{pol}$ or U937 cells, and the CAT activity was assayed.

detectable even at a U937 $\mathrm{p}$ polrecipient cell ratio of $1: 1,000$ (Fig. 5).

To evaluate the time necessary for transcellular activation to take place, donor and recipient cells were separated after various periods of cocultivation. To distinguish between the time required for activation of the LTR and the time necessary for the synthesis and accumulation of CAT, we adhered

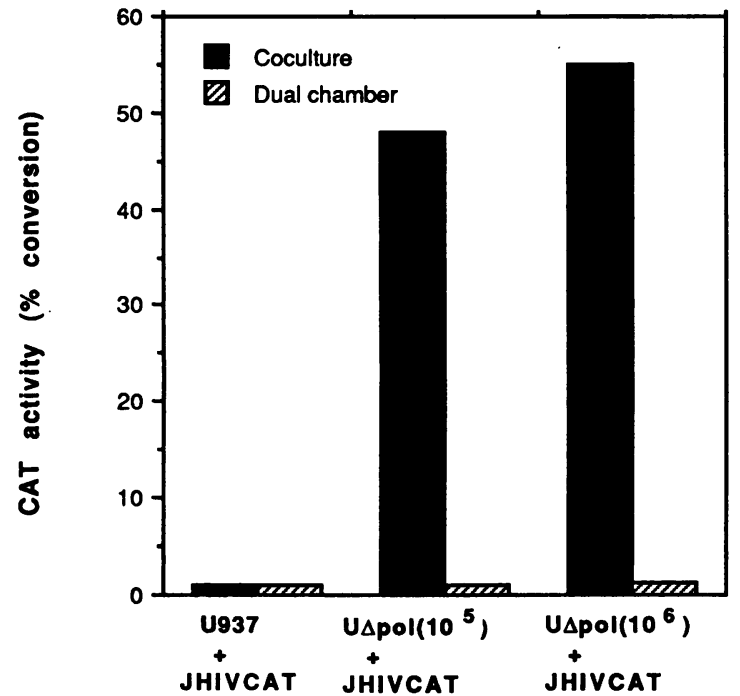

FIG. 4. Transactivation does not occur if $J \Delta$ pol cells are not in contact with the JHIVCAT cells. J $\Delta$ pol cells were separated from the JHIVCAT cells by culturing them in a Millicell insert. In control cultures, the cells were directly cocultured. 


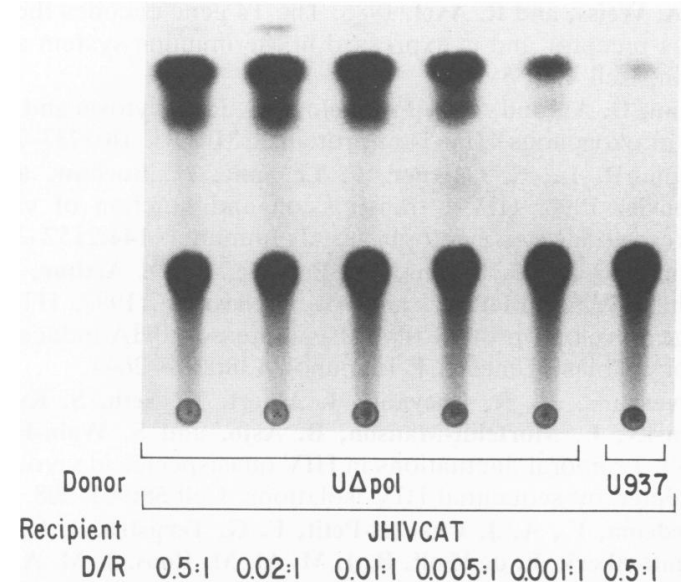

FIG. 5. Transcellular activation occurs over a wide range of donor/recipient (D/R) cell ratios. U937 $\mathrm{pol}$ and JurkatHIVCAT cells were cocultured at the ratios indicated for $48 \mathrm{~h}$. All the cocultures were initiated at the same cell density, with $10^{6} \mathrm{Jur}$ katHIVCAT cells.

U937 $\Delta$ pol cells, which express Fc receptors, to IgG-coated dishes and then added indicator JurkatHIVCAT cells for the specified periods of time (Fig. 6). The JurkatHIVCAT cells were then recovered and cultured overnight to allow for the synthesis of CAT and were assayed for CAT activity. After only $3 \mathrm{~h}$ of coculture, significant transcellular activation was observed. As a control, adherent U937 pol cells were cultured with medium alone. JurkatHIVCAT cells were also cocultured with nontransfected U937 cells to assess whether the interaction of IgG with the Fc receptors of the U937 cells allowed them to induce CAT activity in the JurkatHIVCAT cells independently of the expression of $\mathrm{pHXB} \Delta$ pol. No

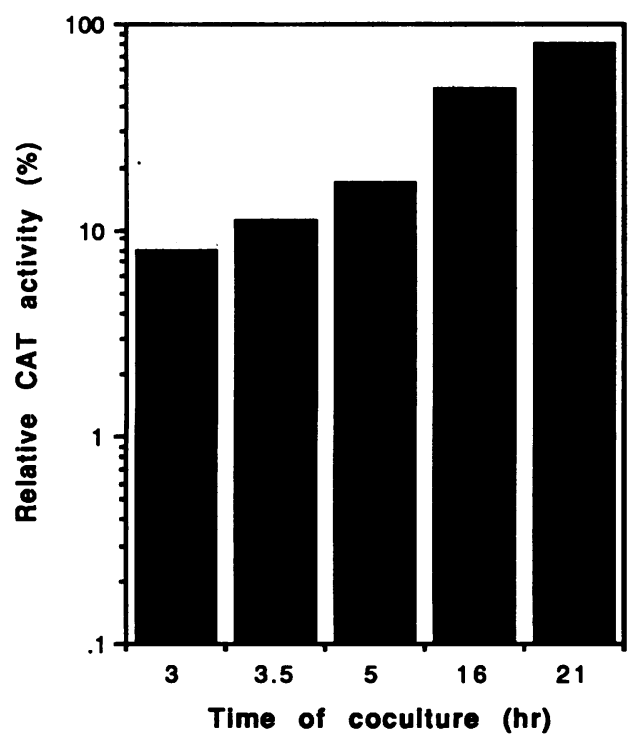

FIG. 6. Kinetics of transactivation. U937 pol cells $\left(3 \times 10^{6}\right)$ were adhered to tissue culture dishes coated with $1 \%$ human IgG, and the nonadherent cells were discarded. JurkatHIVCAT cells $(3 \times$ $\left.10^{6}\right)$ were then added, incubated with the U937Dpol cells for the indicated periods of time, removed, transferred to fresh dishes, and cultured overnight. The results express the relative CAT activity for each time point after subtraction of the corresponding control value. significant increase in CAT activity in either of these control experiments was observed.

\section{DISCUSSION}

We assessed the ability of a small number of HIVexpressing cells to activate the HIV LTR integrated in cocultured, non-HIV-expressing lymphocytes. The plasmids used for these studies expressed the viral genes under the control of the HIV LTR rather than a promoter that would allow abnormally high expression. When cells are simply cocultured for brief periods of time, Tat-mediated transcellular activation is observed. Transcellular activation of the HIV LTR is TAR dependent and is evident in as little as $3 \mathrm{~h}$ of cocultivation and at HIV-expressing-to-target cell ratios as low as 1:1,000. Maximal transactivation occurred at $\mathrm{pHXB} \Delta$ pol-expressing-to-indicator cell ratios of 1:100, suggesting that one HIV-expressing cell can interact with and transactivate multiple neighboring cells.

Transcellular activation of $T$ lymphocytes after coculture with HIV-expressing U937 cells suggests that antigen presentation by HIV-infected macrophages may result in the activation of a latent LTR or alteration of cellular function in the responding $T$ cell. The recent description of efficient transmission of HIV to T lymphocytes by HIV-infected monocytes/macrophages during antigen presentation (4) indicates that this process may play an important role in the dissemination of disease. Subsequent to the activation of the viral LTR in latently infected cells, an amplification loop resulting in greatly enhanced transcription and in the synthesis of viral products is set into motion. Thus, the transfer of only a few Tat molecules from a single activated cell could result in widespread activation of latently infected cells. Tat peptide, added to the medium of cells growing in culture at a concentration of $500 \mathrm{ng} / \mathrm{ml}$, results in a $50 \%$ inhibition of antigen-induced proliferation of lymphocytes (26). Furthermore, after exposure to tat-expressing cells, the ability of $\mathrm{T}$ cells to respond to stimulatory signals (phytohemagglutinin and anti-T3 antibody) is compromised, as measured by the mobilization of $\mathrm{Ca}^{2+}$ in cocultured cells (data not shown). The system described in this report allows us to assess the biological significance of transcellular activation.

The mechanism by which TAR-dependent, transcellular activation occurs is not clear. It has been suggested that Tat might be released into the serum by the lysis of infected cells (13). The kinetics (activation was observed after only $3 \mathrm{~h}$ of cocultivation), the small number of donor cells needed for this effect, and the ability to use stably transfected cell lines which exhibited no appreciable cell death all suggest that cell lysis is not required for transactivation. The absence of transactivation when specific TAR mutants are introduced into the LTR suggests the participation of Tat in stimulating transcription; however, it is difficult to demonstrate the actual transfer of Tat from one cell to another.

The data suggest that there is cell type specificity to the activation, as CAT activity was not induced in U937 recipients. The observation that T cells and U937 cells expressing HIV can activate the LTR in cocultured T cells but not in cocultured U937 cells indicates that the LTR in U937 cells, and possibly all monocytic cells, is functionally inaccessible to transcellular activation by nearby infected cells. This is in contrast to the ability to transactivate the HIV LTR in U937 cells when Tat protein is added to the culture medium (26). These data suggest that an interaction involving a specific accessory molecule present on $T$ cells might play a role in transcellular activation or that the high lysosomal enzyme 
content and proteolytic activity of the monocyte inhibit the activating factor.

Human CD4 expressed on a murine cell does not permit HIV infection or multinucleated syncytium formation (11). The activation of the HIV LTR in murine NIH 3T3 cells cocultured with U937 $\Delta$ pol cells demonstrates that the typically described HIV-mediated syncytium formation is not necessary for transcellular activation.

The potential of HIV-1, as well as other RNA viruses, to change over the course of an infection, is enormous. This allows the accumulation of a large number of defective viruses as the disease progresses because of the introduction of mutations in various points of the viral genome $(7,15)$. Cells transfected with the $\Delta$ pol plasmid constitute a model system for the analysis of the functional consequences of the presence of defective viruses. The data presented here suggest that cells infected with defective viruses may not be benign and may actually contribute significantly to the pathogenesis of the disease.

\section{ACKNOWLEDGMENTS}

We thank J. Sodroski, M. Muesing, and F. Kachanchi for providing plasmids and cell lines and S. C. Silverstein and P. Caldwell for helpful discussions. We gratefully acknowledge the expert technical assistance of Mark Sugden.

This work was supported by National Institutes of Health grants AI26886 and HL43528 to O. K. W.

\section{REFERENCES}

1. Arya, S., C. Guo, S. Josephs, and F. Wong-Staal. 1985. Transactivator gene of HTLV-III. Science 229:69-73.

2. Brake, D. A., C. Debouck, and G. Biesecker. 1990. Identification of an Arg-Gly-Asp (RGD) cell adhesion site in HIV-1 Tat. J. Cell Biol. 111:1275-1281.

3. Cullen, B. 1986. Trans-activation of HIV occurs via a bimodal mechanism. Cell 46:973-982.

4. Dayton, A. I., J. G. Sodroski, C. A. Rosen, W. C. Goh, and W. A. Haseltine. 1986. The trans-activator gene of the human T cell lymphotropic virus type III is required for replication. Cell 44:941-947.

5. Diamond, D. C., B. P. Sleckman, T. Gregory, L. A. Lasky, J. L. Greenstein, and S. J. Burakoff. 1988. Inhibition of CD4+ T cell function by the HIV envelope protein, gp120. J. Immunol. 141:3715-3717

6. Ensoli, B., G. Barillari, S. Z. Salahuddin, R. C. Gallo, and F. Wong-Staal. 1990. Tat protein of HIV-1 stimulates growth of cells derived from Kaposi's sarcoma lesions of AIDS patients. Nature (London) 345:84-86.

7. Goodenow, M., T. Huet, W. Saurin, S. Kwok, J. Sninsky, and S. Wain-Hobson. 1989. HIV-1 isolates are rapidly evolving quasispecies: evidence for viral mixtures and preferred nucleotide substitutions. J. Acquired Immune Defic. Syndr. 2:344-352.

8. Gruters, A. R., F. G. Terpstra, R. De Jong, C. J. M. Van Noesel, R. A. W. Van Lier, and F. Miedema. 1990. Selective loss of T cell functions in different stages of HIV infection. Eur. J. Immunol. 20:1039-1044.

9. Kornfeld, H., W. W. Cruikshank, S. W. Pyle, J. S. Berman, and D. M. Center. 1988. Lymphocyte activation by HIV-1 envelope glycoprotein. Nature (London) 335:445-448.

10. Lifson, J. D., G. R. Reyes, M. S. McGrath, B. S. Stein, and E. G. Engleman. 1986. AIDS retrovirus induced cytopathology: giant cell formation and involvement of CD4 antigen. Science 232: $1123-1127$.

11. Maddon, P. J., A. G. Dalgleish, J. S. McDougal, P. R. Clapham,
R. A. Weiss, and R. Axel. 1986. The T4 gene encodes the AIDS virus receptor and is expressed in the immune system and the brain. Cell 47:333-348.

12. Mann, D. A., and A. D. Frankel. 1991. Endocytosis and targeting of exogenous HIV-1 tat protein. EMBO J. 10:1733-1739.

13. Mann, D. L., S. Gartner, F. Le Sane, H. Buchow, and $M$. Popovic. 1990. HIV-1 transmission and function of virus-infected monocytes/macrophages. J. Immunol. 144:2152-2158.

14. Mann, D. L., F. Lasane, M. Popovic, L. O. Arthur, W. G. Robey, W. A. Blattner, and M. J. Newman. 1987. HTLV-III large envelope protein (gp120) suppresses PHA-induced lymphocyte blastogenesis. J. Immunol. 138:2640-2644.

15. Meyerhans, A., R. Cheynier, J. Albert, M. Seth, S. Kwok, J. Sninsky, L. Morfeldt-Manson, B. Asjo, and S. Wain-Hobson. 1989. Temporal fluctuations in HIV quasispecies in vivo are not reflected by sequential HIV isolations. Cell 58:901-908.

16. Miedema, F., A. J. Chantal-Petit, F. G. Terpstra, J. K. M. E. Schattenkerk, F. de Wolf, B. J. M. Al, M. Roos, J. M. A. Lang, S. A. Danner, J. Goudsmit, and P. T. A. Schellekens. 1988. Immunological abnormalities in HIV-infected asymptomatic homosexual men. HIV affects the immune system before CD4+ T helper cell depletion occurs. J. Clin. Invest. 82:1908-1914.

17. Muesing, M., D. H. Smith, and D. J. Capon. 1987. Regulation of mRNA accumulation by a HIV trans-activator protein. Cell 48:691-701

18. Newstein, M., E. J. Stanbridge, G. Casey, and P. Shank. 1990. Human chromosome 12 encodes a species-specific factor which increases HIV-1 tat-mediated transactivation in rodent cells. J. Virol. 64:4565-4567.

19. Okamoto, T., T. Benter, S. F. Josephs, R. Sadaie, and F. Wong-Staal. 1990. Transcriptional activation from the longterminal repeat of human immunodeficiency virus in vitro. Virology 177:606-614.

20. Oyaizu, N., N. Chirmule, V. S. Kalyanaraman, W. W. Hall, R. A. Good, and S. Pahwa. 1990. HIV-1 envelope glycoprotein gp120 produces immune defects in $\mathrm{CD} 4+\mathrm{T}$ lymphocytes by inhibiting interleukin 2 mRNA. Proc. Natl. Acad. Sci. USA 87:2379-2383.

21. Rosen, C. A., J. G. Sodroski, and W. A. Haseltine. 1985. The location of cis-acting regulatory sequences in the human $\mathrm{T}$ cell lymphotropic virus type III (HTLV-III/LAV). Cell 41:813-823.

22. Schnittman, S. M., M. C. Psallidopoulos, H. C. Lane, L. Thompson, M. Baseler, F. Massari, C. H. Fox, N. P. Salzman, and A. S. Fauci. 1989. The reservoir for HIV-1 in human peripheral blood is a $\mathrm{T}$ cell that maintains expression of CD4. Science 245:305-308.

23. Shearer, G. M., D. C. Bernstein, K. S. Tung, C. S. Via, R. Redfield, S. Z. Salahuddin, and R. C. Gallo. 1986. A model for the selective loss of major histocompatibility complex selfrestricted $\mathrm{T}$ cell immune responses during the development of AIDS. J. Immunol. 137:2514-2521.

24. Sodroski, J., W. C. Goh, C. Rosen, K. Campbell, and W. Haseltine. 1986. A second post-transcriptional trans-activator gene required for HTLV-III replication. Nature (London) 322: $470-474$.

25. Sodroski, J., R. Patarca, C. A. Rosen, F. Wong-Staal, and W. Haseltine. 1985. Location of the trans-activating region on the genome of HTLV-III. Science 229:74-77.

26. Viscidi, R. P., K. Mayur, H. M. Lederman, and A. D. Frankel. 1989. Inhibition of antigen-induced lymphocyte proliferation by Tat protein from HIV-1. Science 246:1606-1608.

27. Wahl, L. M., M. L. Corcoran, S. W. Pyle, L. O. Arthur, A. Harel-Bellan, and W. L. Farrar. 1989. HIV-1 and its envelope glycoprotein down-regulate chemotactic ligand receptors and chemotactic function of peripheral blood monocytes. Proc. Natl. Acad. Sci. USA 86:621-625. 